Research Article in Physics

\title{
The investigation on the AC to DC voltage multiplier
}

\section{Taige Chen ${ }^{1, *}$}

${ }^{1}$ High School Attached to Northeast Normal University, Changchun, Jilin 410006, China

* Correspondence: 2655230058@qq.com

(Received: 10/25/2021; Accepted: 12/07/2021; Published: 12/20/2021)

DOI: https://doi.org/10.37906/isteamc.2021.10

\begin{abstract}
This paper investigates the topic of voltage multiplication, which converts a low AC voltage source to a high DC voltage source. Several designs are evaluated, such as the voltage doubler, the voltage tripler, and the voltage quadrupler. It is discovered that the input frequency and the capacitance do not affect the output voltage. This design can be extended to any integer multiples of the input voltage.
\end{abstract}

Keywords: voltage multiplier, AC, DC, circuit

\section{Introduction}

In many modern-day equipment, a high DC voltage source is needed, but the most common voltage source is the AC voltage source. Therefore, it can be useful to design a circuit that can convert the AC voltage source to a DC one but with a higher voltage. With this circuit, the laboratory researchers do not have to worry about the lack of DC voltage source, which is useful for advanced experiments such as particle accelerators. There are already some investigation on the AC-DC multiplier (Zaid et al., 2021). However, these designs can be extremely complex and difficult to create. Hence, the goal of this paper is to investigate a simpler setup. The paper "Design and Implementation of an AC to High DC Voltage Generation Circuit Using Voltage Multiplier" (Md. Mohasin Siddique February, 2019) serves as the basis of this paper, detailing the principles that makes the voltage change possible. It also changes the small AC voltage into the high DC voltage.

This paper investigates how much voltage multiplication will be obtained experimentally from each stage. This paper will examine the two stages of the voltage multiplication and determine whether the voltage multiplication depend on capacitance value and the input frequency or not.

\section{Research Design, Data collection and analysis Methods, Materials}

Through the falstad circuit simulation website (http://www.falstad.com/circuit/), we were able to design the circuit which contains capacitors and diodes for the voltage multiplication. Firstly, we want to design a circuit which contains only two diodes and two capacitors, which we call it the first stage. During this stage, what we want to do is to design an optimal circuit which can maximum the output voltage at a certain input voltage. If there are multiple research questions or if multiple studies are needed to answer a single research question, the paper should include subsections of methods and designs for each of the studies to be conducted. 
Study 1: Investigation of voltage doubler

Figure 1: The voltage doubler circuit

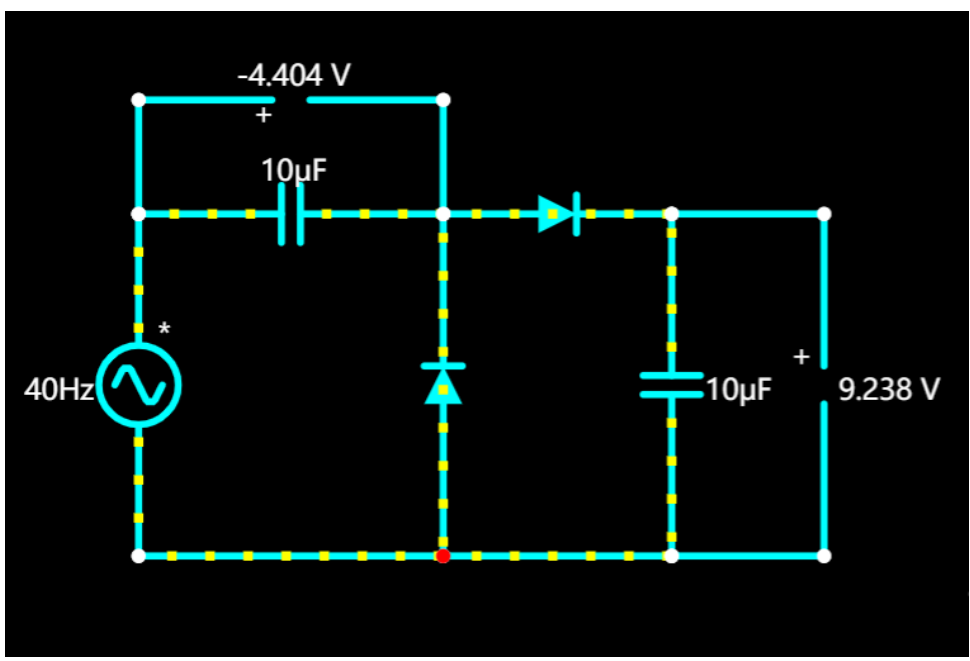

From Figure 1, we can see that the voltage value of the two capacitors. In this experiment, we have set the input voltage, the voltage of the $\mathrm{AC}$ voltage source, to $5 \mathrm{~V}$. However, the capacitor that is located on the right side has a voltage of $9.238 \mathrm{~V}$, which means that the voltage is almost doubled. The voltage of the capacitor on the right side is less than $10 \mathrm{~V}$, the double of initial input voltage, which is due to the nonzero diode resistance. We may decompose this circuit into two identical circuits which we will show out in the figures below.

\section{Figure 2: Part 1 of the voltage doubler circuit}

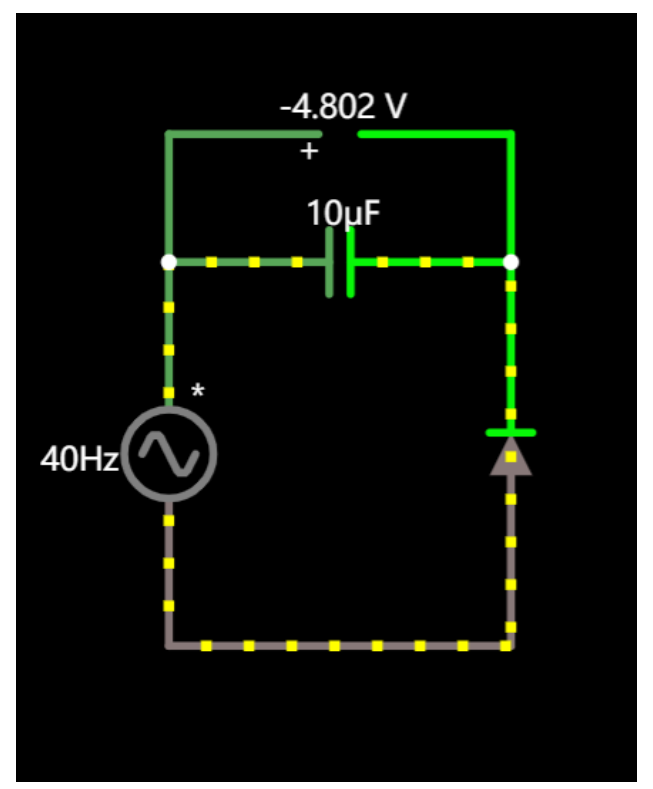


Figure 3: Part 2 of the voltage doubler circuit

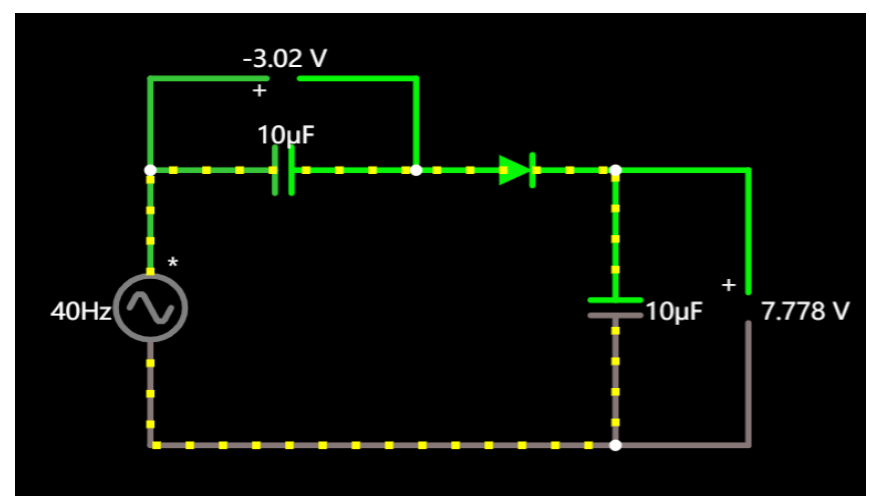

The whole circuit can be seen as the superposition of the two circuits above. In Figure 2, the capacitor charges to $4.802 \mathrm{~V}$ on the negative half cycle of AC input (the counterclockwise). Due to the diode, the AC source can only transmit current in one path rather than two paths. Another effect of the existing diode is its resistance which cause the voltage charged by the capacitor dropped from $5 \mathrm{~V}$ to $4.802 \mathrm{~V}$. Therefore, if this circuit is represented in reality, the actual voltage output will be smaller than the double one.

During the positive half cycle, shown in Figure 3, the diode in the middle of the circuit allows for current passage because it is reverse biased. Now the two capacitors are all in the series with the AC voltage input. We can see that the AC voltage input and the capacitor on the left-hand side are in series, which means that the voltage can be added up to find the voltage of the capacitors on the right-hand side. Thus, that capacitor on the right-hand side has a total of $10 \mathrm{~V}$ experimentally, $5 \mathrm{~V}$ from the AC source and $5 \mathrm{~V}$ from the other capacitor.

\section{Study 2: The investigation of different factors that might affect the voltage multipler}

Figure 4,5,6: The voltage doubler circuits with different capacitance

Figure 4

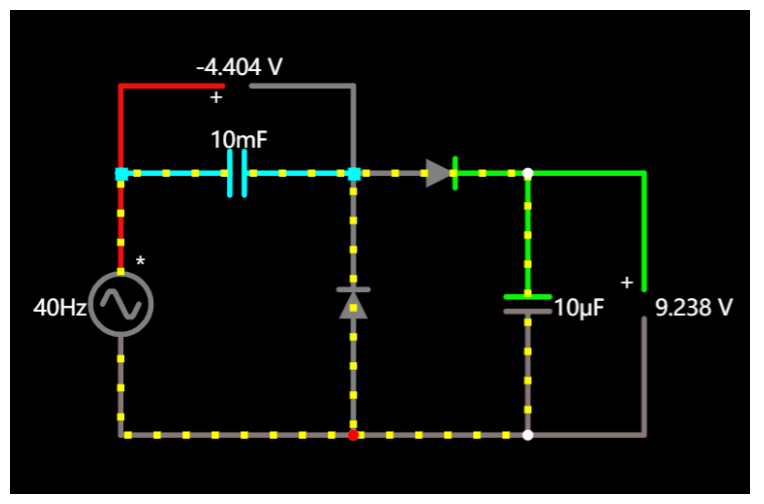

Figure 5

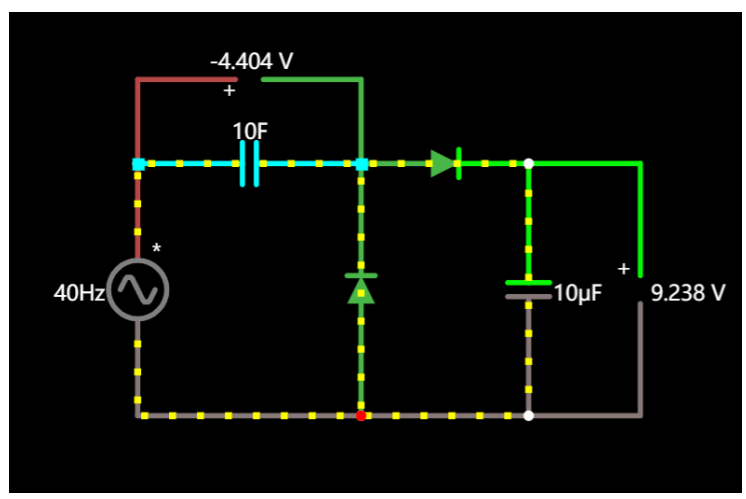




\section{Figure 6}

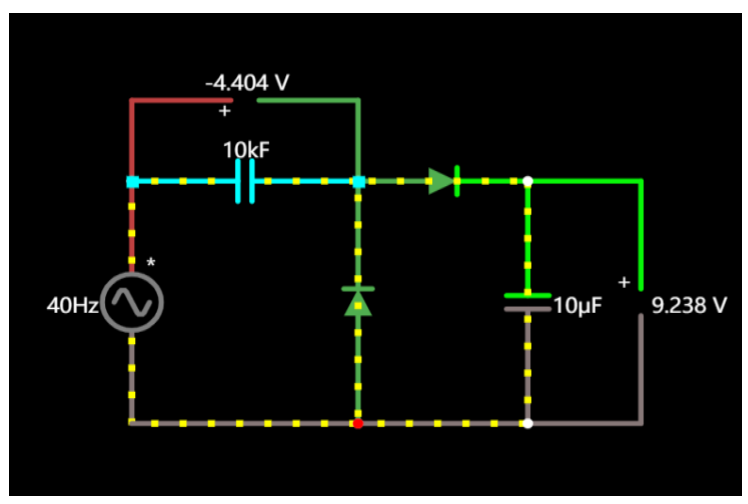

In the three figures above, the capacitance of the capacitor is changed to see whether the value of capacitance influences the output voltage. We have changed the capacitance to the following three values: $10 \mathrm{mF}, 10 \mathrm{~F}$, and $10 \mathrm{kF}$. We have set the interval of 1000 multiple in order to make the influences of the output voltage clear. However, as we can see in the graph, there are no changes in the output voltage as we change the value of one capacitor. Therefore, we can conclude that the values of the capacitor will not influence the output at all.

For the next step, we will try to change the frequency of the input AC voltage. In this case, we will investigate whether changing the frequency of input $\mathrm{AC}$ voltage will influence the output voltage.

Figure 7, 8, 9: The voltage doubler circuits with different frequency of the input voltage
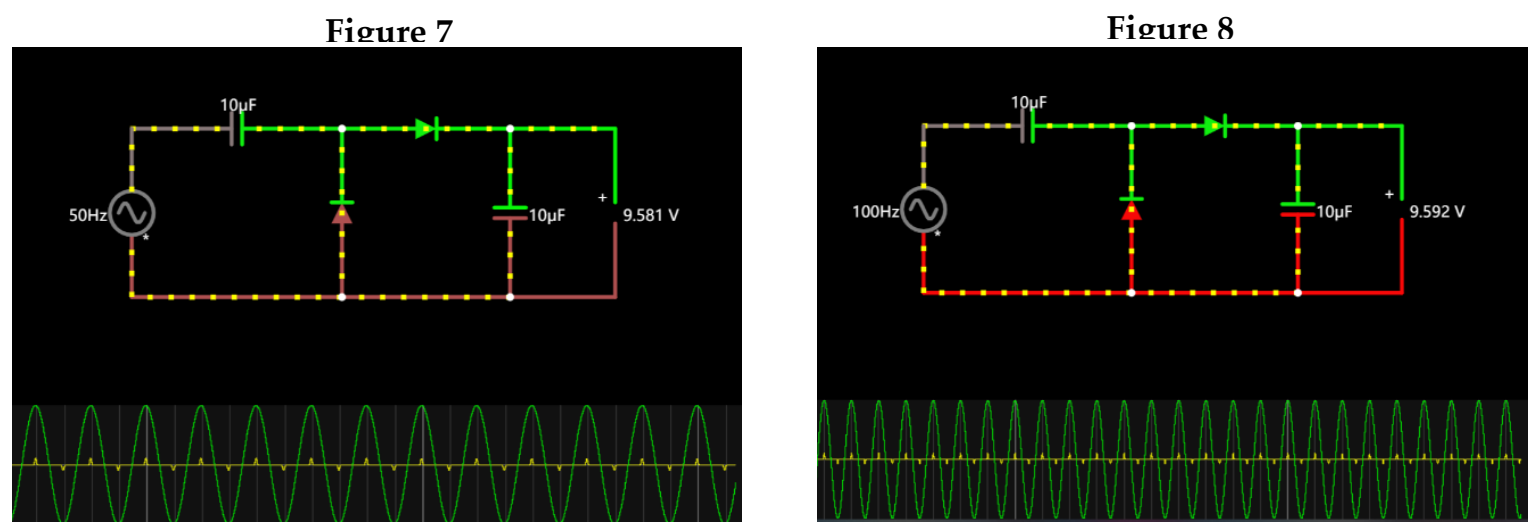

Figure 9

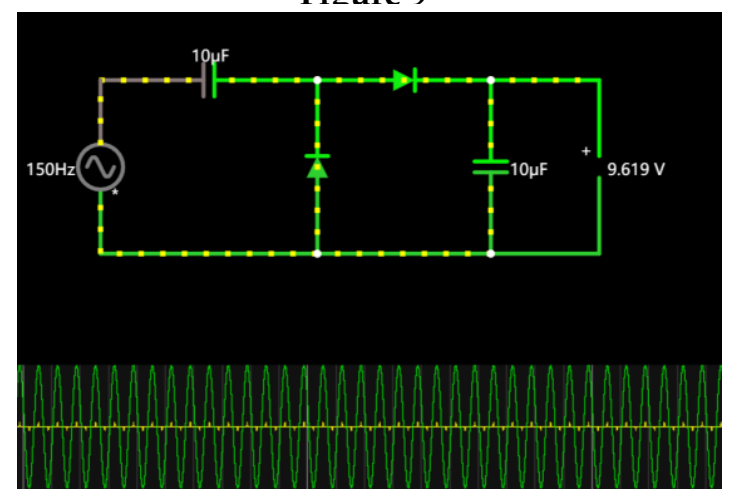


In the three figures above, the three oscilloscope signals are all showing about the trends between the voltage across the capacitor and time under different input frequencies. Firstly, we can see that there are no changes on the final output voltage with the changes on the input frequency. However, frequency is the number of cycles in a unit time, which means that the frequency affects the rate of change in the direction of the input voltage. Therefore, as the frequency of the input voltage increases, the frequency of the output voltage also increases proportionally.

As a result, based on the investigation above, there is no obvious connection between the input frequency and the capacitance of the capacitors with the final output voltage, which means that in this circuit, the only things that affect the final output voltage are the initial input voltage and the sets of diode and capacitors. Therefore, we will continue to design and advance this circuit in the section 2 into higher multiple of initial voltage.

\section{Study 3: The investigation of voltage multipler of higher multiples}

In section 2, we will try to create different types of voltage multiplier in order to increase the multiple of the input voltage. In this case, people can simply change the structure of the multiplier to change the output voltage. In section 1, we have designed the double output voltage circuit, known as the voltage doubler. Therefore, in this section, we will start with the voltage tripler and the voltage quadrupler.

\section{Design of voltage tripler:}

Figure 10: The voltage tripler

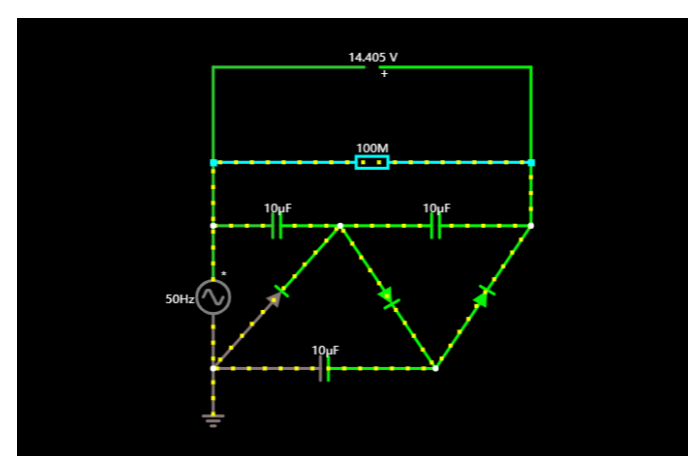

In the design of voltage tripler, we simplify the structure of the voltage doubler, and added a resistor to the circuit to measure the output voltage and stabilize the circuit.

As we can see in the figures, the circuit has three sets of diode and capacitor, which create a triple multiple of the initial input voltage. The trend is clear that with the increase numbers of sets of diode and capacitor, the higher the multiple of initial input voltage can be. Therefore, we can go further to try for the voltage quadrupler to see whether the trend fits my assumption. 


\section{Design of voltage quadrupler:}

Figure 11: The voltage quadrupler

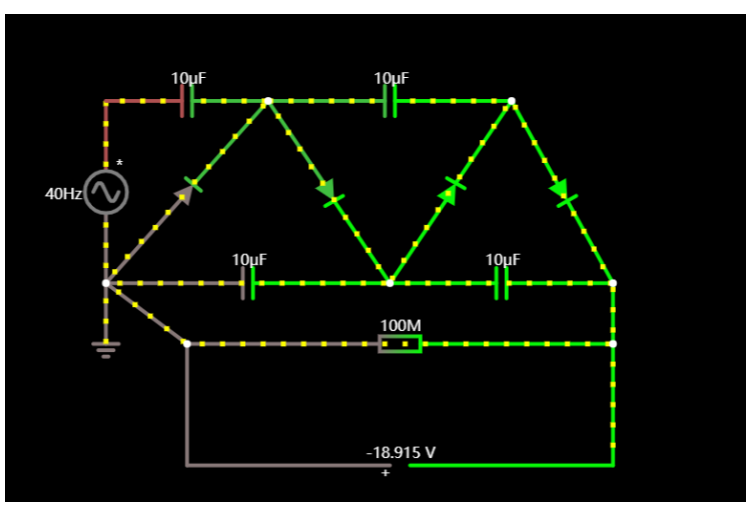

We added the fourth set of diode and capacitor into the circuit. In this circuit, the final output only reaches approximately $19 \mathrm{~V}$, slightly less than the desired $20 \mathrm{~V}$, which means that there is some voltage loss in the whole circuit.

Afterwards, one can simply add more sets of diode and capacitor into the circuit to get any integer multiples of the input voltage. However, there is voltage loss in any sets of circuits, which means that these circuits imitate the real situation of the circuits. To improve this situation, one can lower the resistance of the wires or the diodes.

\section{Conclusions}

In conclusion, based on my observation, we were able to increase the final output voltage step by step by adding sets of diode and capacitor. On the other hand, the input frequency and the capacitance do not affect the final output voltage, which means that in actual designs, people have no need to worry about the capacitor and the initial frequency.

\section{Reference:}

http://www.falstad.com/circuit/

Mohammad Zaid, Shahrukh Khan, Marif Daula Siddique, Adil Sarwar, Javed Ahmad, Zeeshan Sarwer, Atif Iqbal (2021 March 25). A transformerless high gain dc-dc boost converter with reduced voltage stress.

Design and Implementation of an AC to High DC Voltage Generation Circuit Using Voltage Multiplier. (Md. Mohasin Siddique, 2019) 